\title{
Low Complexity Adaptation for Reconfigurable Intelligent Surface-Based MIMO Systems
}

\author{
Zehra Yigit, Student Member, IEEE, Ertugrul Basar, Senior Member, IEEE, \\ and Ibrahim Altunbas, Senior Member, IEEE.
}

\begin{abstract}
Reconfigurable intelligent surface (RIS)-based transmission technology offers a promising solution to enhance wireless communication performance cost-effectively through properly adjusting the parameters of a large number of passive reflecting elements. This letter proposes a cosine similarity theorem-based low-complexity algorithm for adapting the phase shifts of an RIS that assists a multiple-input multiple-output (MIMO) transmission system. A semi-analytical probabilistic approach is developed to derive the theoretical average bit error probability (ABEP) of the system. Furthermore, the validity of the theoretical analysis is supported through extensive computer simulations.
\end{abstract}

Index Terms-Reconfigurable intelligent surfaces (RISs), multiple-input multiple-output (MIMO), cosine similarity theorem.

口

\section{INTRODUCTION}

$\mathbf{T}$ HE fifth generation (5G) wireless communication technology promises an explosive growth on data rate, massive connectivity and latency performance. To achieve these goals, various transmission technologies have been developed in recent years. Massive multiple-input multiple-output (MIMO) and milimeter wave (mmWave) communication systems are considered as some of the prominent candidates among these technologies. On the other hand, to meet this challenge beyond $5 \mathrm{G}$ requirements, utilization of an increasing number of multi-antenna systems has raised strong concerns about the energy efficiency and hardware cost of large-scale MIMO systems.

Recently, reconfigurable intelligent surface (RIS)-assisted communication technology has been considered as a promising solution to overcome the energy efficiency related issues of future wireless networks [1]-[3]. An RIS is a planar metasurface that consists of a number of low-cost passive reflecting elements, each of which smartly induces an independent phase shift to modify the propagation environment in more favorable way for the communication performance [4].

The unprecedented potential of RISs on the signal quality of a communication system has led researchers to largely consider the RIS technology in various frontiers. In one of the early studies [3], the error performance of an RIS aided singleinput single-output (SISO) system is investigated through a

Z. Yigit and Ibrahim Altunbas are with the Department of Electronics and Communication Engineering, Istanbul Technical University, Maslak 34469, Istanbul, Turkey. E-mail: yigitz@itu.edu.tr, ibraltunbas@itu.edu.tr.

E. Basar is with the Communications Research and Innovation Laboratory (CoreLab), Department of Electrical and Electronics Engineering, Koç University, Sariyer 34450, Istanbul, Turkey. E-mail: ebasar@ku.edu.tr

This work was supported by the Scientific and Technological Research Council of Turkey (TÜBİTAK) under grant no 117E869. mathematical framework. Also, in [5], [6], to improve the spectral efficiency, the RIS aided index modulation (IM) systems have been proposed. Later, RISs are considered for multiuser systems to enhance the energy efficiency [7] and to maximize the signal-interference-noise ratio (SINR) [2], [8], [9]. Even more recently, to facilitate practical implementation of the RIS aided systems, new path loss models [10], [11], open-source channel models [12], [13] and a practical phase shift model [14] have been developed.

Notably, most of the existing RIS-aided designs developed for multiple-input single-output (MISO) [2], [7], [8], [15] and MIMO systems [1], [9] exploit computationally intensive and complex algorithms. Specifically, no single study exists performing a statistical analysis on the theoretical bit error rate (BER) performance of RIS-aided multi-antenna systems.

In this letter, an efficient low-complexity algorithm, which is based on cosine similarity theorem [16] and adapts the phase shift of each reflecting elements, is proposed for RISaided MIMO (RIS-MIMO) and RIS-aided spatial modulation (RIS-SM) schemes in which the transmission principles of classical MIMO and classical SM [5], [17] are considered, respectively. Moreover, a semi-analytical probabilistic model is developed to derive the average bit error probability (ABEP) of the proposed RIS-MIMO and RIS-SM schemes. Furthermore, through the comprehensive computer simulations, the BER performance of the RIS-aided MIMO systems with the proposed algorithm is investigated for the various conditions including perfect/imperfect channel estimation, continous/discrete phase reflection and path loss effect.

The rest of this letter is structured as follows. In Section II, the system model of the proposed RIS-aided MIMO transmission systems is presented. The theoretical performance analysis of the system is carried out in Section III. Computer simulation results are described in Section IV and the paper is concluded in Section $\mathrm{V}$.

\section{SySTEM MODEL}

In this section, the concept of the proposed low-complexity algorithm and the system models of the RIS-MIMO and RIS-

\footnotetext{
${ }^{1}$ Notation: Bold lowercase and uppercase letters are used for vectors and matrices, respectively. $(\cdot)^{\mathrm{T}},(\cdot)^{\mathrm{H}}$ and $\|\cdot\|$ stand for transposition, Hermitian transposition and Euclidean/Frobenious norm operators, respectively. $\operatorname{vec}(\cdot)$ denotes vectorization operator and $\operatorname{det}(\cdot)$ symbolizes determinant of a matrix. The Kronecker product and Euclidean inner product of two vectors are denoted by $\otimes$ and $\langle\cdot, \cdot\rangle$, respectively. $\mathbb{C}^{a \times b}$ represents the set of $a \times b$ dimensional matrices while $\operatorname{diag}\{\cdot\}$ symbolizes a square diagonal matrix. $\mathcal{C N}\left(\mu, \sigma^{2}\right)$ denotes the complex Gaussian distribution of a random variable with $\mu$ mean and $\sigma^{2}$ variance. $\mathcal{O}(\cdot)$ and $P(\cdot)$ stand for the big $\mathcal{O}$ notation and probability of an event, respectively. $\mathbf{I}_{n}$ denotes $n \times n$ identity matrix while $\mathbf{1}$ represents all-ones column vector.
} 


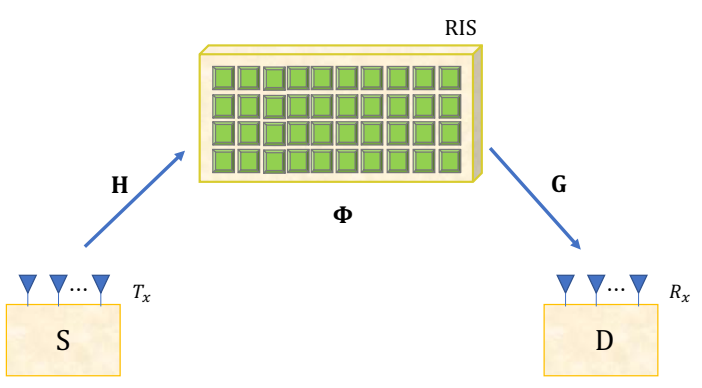

Fig. 1. RIS aided MIMO Systems

SM schemes are introduced. In the proposed systems, the transmitter and the receiver are assumed to be equipped with $T_{x}$ and $R_{x}$ antennas, respectively, as shown in Fig. 1. In addition, an RIS with $N$ passive reflecting elements is used to improve the communication performance by appropriately adjusting phase shift of each reflecting element. Consider $\mathbf{H} \in \mathbb{C}^{N \times T_{x}}$ and $\mathbf{G} \in \mathbb{C}^{N \times R_{x}}$ as the matrices of uncorrelated Rayleigh fading channel from the transmitter to the RIS, and from the RIS to the receiver, respectively, whose elements are independent and identically distributed (i.i.d.) and follow $\mathcal{C N}(0,1)$ distribution. On the other hand, $\Phi \in \mathbb{C}^{N \times N}$ stands for the matrix of RIS reflection coefficients with $\mathbf{\Phi}=\operatorname{diag}\left\{\beta_{1} e^{j \phi_{1}}, \beta_{2} e^{j \phi_{2}}, \ldots, \beta_{N} e^{j \phi_{N}}\right\}$, where $\phi_{i} \in[-\pi, \pi]$ is phase shift and $\beta_{i} \in(0,1]$ is the amplitude reflection coefficient of the $i$ th reflecting element, for $i \in\{1,2, \ldots, N\}$. However, for the sake of simplicity, we set $\beta_{i}=1$. Therefore, the composite MIMO channel matrix $\mathbf{C} \in \mathbb{C}^{R_{x} \times T_{x}}$ from the transmitter to the receiver becomes $\mathbf{C}=\mathbf{G}^{\mathrm{H}} \mathbf{\Phi} \mathbf{H}$. In the proposed systems, perfect channel state information (CSI) of all channels are available at all nodes and quasi-static block fading channels are assumed.

\section{A. Proposed Algorithm}

In this subsection, we develop a low-complexity algorithm to maximize the average received signal-noise ratio (SNR) of the RIS aided MIMO systems, which results in maximizing the overall channel gain of the system by arranging the phase shift of each reflecting element. Then, using $\mathbf{C}=\mathbf{G}^{\mathrm{H}} \mathbf{\Phi} \mathbf{H}=$ $\sum_{i=1}^{N} \mathbf{g}_{i}^{H} e^{j \phi_{i}} \mathbf{h}_{i}$, our problem is formulated as

$$
\begin{aligned}
& \max _{\phi_{i}}\|\mathbf{C}\|=\max _{\phi_{i}}\left\|\sum_{i=1}^{N} \mathbf{g}_{i}^{H} e^{j \phi_{i}} \mathbf{h}_{i}\right\| \\
& \text { s.t. }\left|e^{j \phi_{i}}\right|=1
\end{aligned}
$$

where $\mathbf{g}_{i}^{\mathrm{H}}$ and $\mathbf{h}_{i}$ stand for the $i$ th column and $i$ th row of $\mathbf{G}^{\mathrm{H}}$ and $\mathbf{H}$, respectively. Although the maximization problem in (11) is non-convex due to the constraint $\phi_{i} \in[-\pi, \pi]$, the achievable channel gain can be upper bounded as

$$
\|\mathbf{C}\|=\left\|\sum_{i=1}^{N} \mathbf{g}_{i}^{H} e^{j \phi_{i}} \mathbf{h}_{i}\right\| \leq \sum_{i=1}^{N}\left\|\mathbf{g}_{i}^{\mathrm{H}}\right\|\left\|\mathbf{h}_{i}\right\|
$$

Then, exploiting (2), the maximum achievable gain of the component at the $k$ th row and the $l$ th column of $\mathbf{C}$, shown by $c_{k, l}$, can be given as

$$
\left|c_{k, l}\right|=\left|\sum_{i=1}^{N} g_{k, i} e^{j \phi_{i}} h_{i, l}\right| \leq \sum_{i=1}^{N}\left|g_{k, i}\right|\left|h_{i, l}\right|
$$

where $g_{k, i}$ and $h_{i, l}$ are the $k$ th and $l$ th components of the channel vectors $\mathbf{g}_{i}^{\mathrm{H}}$ and $\mathbf{h}_{i}$, respectively, for $k \in\left\{1,2, \ldots, R_{x}\right\}$

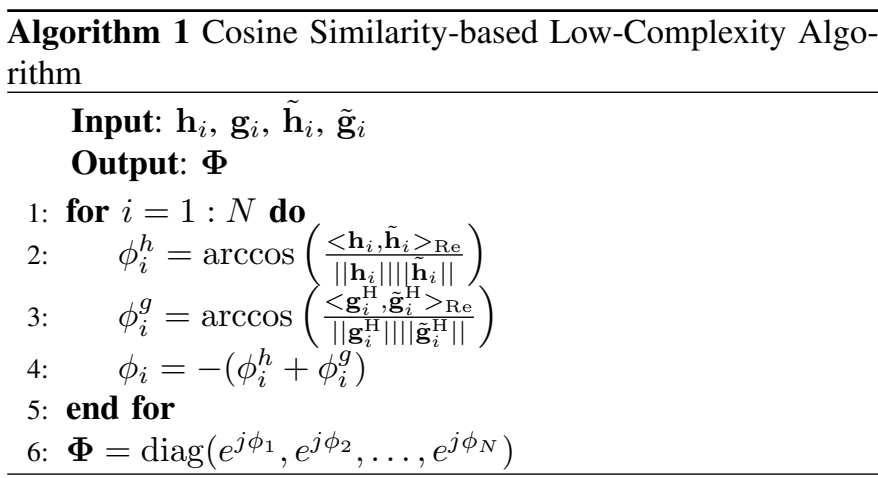

and $l \in\left\{1,2, \ldots, T_{x}\right\}$. It is quite obvious that there exists an optimum $\boldsymbol{\Phi}$ matrix that satisfies (3) with an equality to achieve the maximum channel gain. However, due to the nonconvex constraints, it is difficult to find an optimal solution for this problem in a computationally efficient and robust manner. Moreover, this challenge increases when a multiantenna system is considered at both the transmitter and the receiver. Due to these limitations, we develop an efficient suboptimal solution using the cosine similarity theorem [16], where the angle between $\mathbf{u}$ and $\mathbf{v}$ vectors is calculated, using their inner product and magnitudes [16], as

$$
\cos (\mathbf{u}, \mathbf{v})=\frac{<\mathbf{u}, \mathbf{v}>}{\|\mathbf{u}\|\|\mathbf{v}\|} .
$$

In the proposed algorithm, to maximize the individual gain of each $c_{k, l}$ component (3) the phase shift $\phi_{i}$ is adjusted to approximate the complex channel vectors $\mathbf{h}_{i}$ and $\mathbf{g}_{i}^{\mathrm{H}}$ to their component-wise absolute vectors $\tilde{\mathbf{h}}_{i}=\left[\left|h_{i, 1}\right|,\left|h_{i, 2}\right|, \ldots\left|h_{i, T_{x}}\right|\right]$ and $\tilde{\mathbf{g}}_{i}^{\mathrm{H}}=\left[\left|g_{1, i}\right|,\left|g_{2, i}\right|, \ldots\left|g_{R_{x}, i}\right|\right]^{\mathrm{H}}$, respectively. Therefore, in Algorithm 1, real $\phi_{i}^{h}$ and $\phi_{i}^{g}$ angles are calculated in order to measure the cosine similarity between the vectors $\mathbf{h}_{i}$ and $\tilde{\mathbf{h}}_{i}$ and $\mathbf{g}_{i}^{\mathrm{H}}$ and $\tilde{\mathbf{g}}_{i}^{\mathrm{H}}$, respectively. Then, for the $i$ th reflecting element, the overall phase shift $\phi_{i}$ is determined as $\phi_{i}=-\left(\phi_{i}^{h}+\phi_{i}^{g}\right)$ and the overall reflection matrix $\boldsymbol{\Phi}$ is obtained accordingly.

Let us present the concept of the proposed algorithm by an example. Consider an RIS-aided MIMO system, with $T_{x}=2$, $R_{x}=3$ ve $N=2$ whose composite channel matrix $\mathbf{C}=$ $\mathbf{G}^{\mathrm{H}} \mathbf{\Phi} \mathbf{H}$ is constructed as

$$
\mathbf{C}=\left[\begin{array}{ll}
g_{11} & g_{12} \\
g_{21} & g_{22} \\
g_{31} & g_{32}
\end{array}\right]\left[\begin{array}{cc}
e^{j \phi_{1}} & 0 \\
0 & e^{j \phi_{2}}
\end{array}\right]\left[\begin{array}{ll}
h_{11} & h_{12} \\
h_{21} & h_{22}
\end{array}\right]
$$

which can be rewritten, in the form of $\mathbf{C}=\sum_{i=1}^{N} \mathbf{g}_{i}^{H} e^{j \phi_{i}} \mathbf{h}_{i}$, as

$$
\mathbf{C}=\left[\begin{array}{l}
g_{11} \\
g_{21} \\
g_{31}
\end{array}\right] e^{j \phi_{1}}\left[\begin{array}{ll}
h_{11} & h_{12}
\end{array}\right]+\left[\begin{array}{l}
g_{12} \\
g_{22} \\
g_{32}
\end{array}\right] e^{j \phi_{2}}\left[\begin{array}{ll}
h_{21} & h_{22}
\end{array}\right] .
$$

Since the phase shift $\phi_{i}$ only affects $\mathbf{g}_{i}^{\mathrm{H}}$ and $\mathbf{h}_{i}$ vectors, instead of jointly adjusting all phases, each $\phi_{i}$ can be individually determined in a more computationally efficient manner. Therefore, in the proposed algorithm, considering 3 , we singly determine each $\phi_{i}$ to improve the overall channel gain. For this aim, $\phi_{i}$ is properly adjusted to make the channel vectors $\mathbf{h}_{i}$ and $\mathbf{g}_{i}^{\mathrm{H}}$ approximate to their component-wise absolute vectors $\tilde{\mathbf{h}}_{i}=\left[\left|h_{i, 1}\right|,\left|h_{i, 2}\right|\right]$, and $\tilde{\mathbf{g}}_{i}^{\mathrm{H}}=\left[\left|g_{1, i}\right|,\left|g_{2, i}\right|,\left|g_{3, i}\right|\right]^{\mathrm{H}}$, 
respectively. Therefore, as given in Algorithm 1, the real angles $\phi_{i}^{h}$ and $\phi_{i}^{g}$, which respectively measure the similarities between the vectors $\mathbf{h}_{i}$ and $\tilde{\mathbf{h}}_{i}$, and the vectors $\mathbf{g}_{i}^{\mathrm{H}}$ and $\tilde{\mathbf{g}}_{i}^{\mathrm{H}}$, are calculated using the cosine similarity theorem [16]. Then, the effective phase shift of $i$ th reflecting elements is set to $\phi_{i}=-\left(\phi_{i}^{h}+\phi_{i}^{g}\right)$. After $N$ repetitions are performed, the overall reflection matrix $\boldsymbol{\Phi}$ is constructed.

The required complexity to perform this algorithm is $\mathcal{O}\left(6 N\left(T_{x}+R_{x}\right)\right)$ in terms of real multiplications (RMs). It is worth noting that when a SISO system $\left(T_{x}=R_{x}=1\right)$ is considered, this algorithm satisfies (3) with an equality that achieves the optimum channel gain in [3].

\section{B. RIS-MIMO Scheme}

In the proposed RIS-MIMO scheme with $T_{x}$ transmit antennas, $R_{x}$ receive antennas and $N$ reflecting elements, a classical MIMO transmission principle is applied and the reflection parameters are determined by performing the proposed lowcomplexity algorithm to improve the overall channel gain. Let $\mathbf{x}=\left[x_{1}, x_{2}, \ldots, x_{T_{x}}\right]^{\mathrm{T}} \in \mathbb{C}^{T_{x} \times 1}$ be the transmitted signal vector of the RIS-MIMO scheme, where $x_{j}$ denotes an $M$-ary phase shift keying/quadrature amplitude modulation (PSK/QAM) symbol transmitted through $j$ th transmit antenna, for $j \in\left\{1,2, \ldots, T_{x}\right\}$. Then, the received signal vector $\mathbf{y} \in \mathbb{C}^{R_{x} \times 1}$ becomes

$$
\begin{aligned}
\mathbf{y} & =\mathbf{G}^{\mathrm{H}} \mathbf{\Phi} \mathbf{H} \mathbf{x}+\mathbf{n} \\
& =\mathbf{C} \mathbf{x}+\mathbf{n}
\end{aligned}
$$

where $\mathbf{n} \in \mathbb{C}^{R_{x} \times 1}$ is the vector of additive white Gaussian noise samples whose elements are i.i.d. and follow $\mathcal{C N}\left(0, N_{0}\right)$ distribution.

\section{RIS-SM Scheme}

In this subsection, the system model of the RIS-SM scheme, where an RIS-aided MIMO scheme applying the traditional SM transmission principle [17] is presented. Unlike traditional SM [17], in the proposed RIS-SM scheme, the transmitted signal quality is significantly improved by the RIS, whose each reflection parameter is arranged using the proposed lowcomplexity algorithm.

In RIS-SM, one of the $T_{x}$ transmit antenna is activated and a modulated symbol $s$ from the $M$-PSK constellation is transmitted through this active antenna. Since the remaining transmit antennas are deactivated, the signal vector is determined as $\mathbf{x}=\left[\begin{array}{lllllll}0 & \ldots & 0 & s & 0 & \ldots & 0\end{array}\right]^{\mathrm{T}}$ and transmitted through the overall channel matrix $\mathbf{C}$ as given in (7).

Note that in the proposed RIS-SM scheme, the knowledge of the active antenna index is not provided to the RIS.

At the receiver of both RIS-MIMO and RIS-SM schemes, to obtain the best BER performance, a maximum likelihood (ML) detector is considered, and the transmit RIS-SM and RIS-MIMO vectors are detected by considering all possible $\mathbf{x}$ realizations as follows

$$
\hat{\mathbf{x}}=\arg \min _{\mathbf{x}}\|\mathbf{y}-\mathbf{C x}\|^{2} .
$$

\section{Performance Analysis}

In this section, the theoretical BER performance of the proposed RIS-MIMO and RIS-SM schemes is evaluated by performing a semi-analytical probabilistic approach.

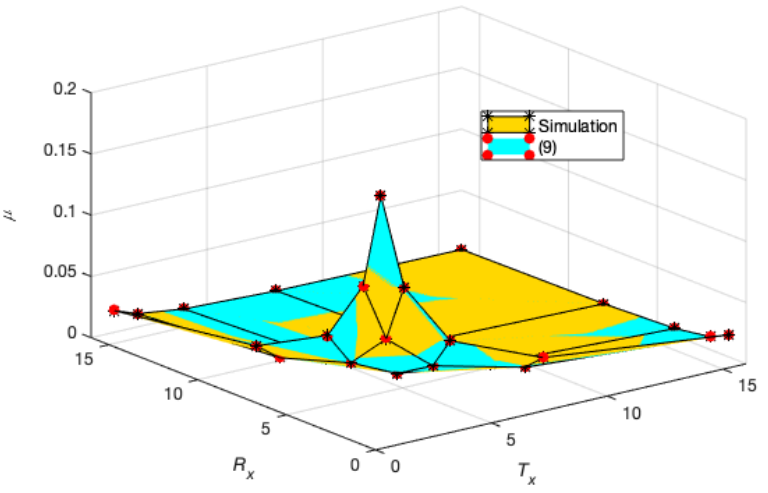

Fig. 2. Comparison of the mean $\mu$ (9) with the Monte-Carlo simulations for different $T_{x}$ ve $R_{x}$ antennas.

\section{A. Numerical Analysis}

As given in Algorithm 1, since the proposed algorithm relates the phase shifts of the reflecting elements with the channel statistics, the reflection matrix $\boldsymbol{\Phi}$ is directly correlated with the channel matrices $\mathbf{H}$ and $\mathbf{G}$. Therefore, the distribution of the composite channel matrix $\mathbf{C}$ could not be derived through a fully analytic approach. As a result, we resort to a comprehensive numerical analysis in order to obtain the statistics of the channel matrix $\mathbf{C}$.

As it is stated in the previous section, the elements of the channel matrices $\mathbf{H}$ and $\mathbf{G}$ are i.i.d. and follow $\mathcal{C N}(0,1)$ distribution. Our comprehensive numerical analysis, which performs $10^{6}$ Monte-Carlo trials for each $R_{x} \times T_{x}$ configuration, indicates that the elements of the composite $\mathbf{C}$ matrix are complex Gaussian random variables with $\mathcal{C N}(N \mu, N)$ distribution, where $\mu$ is numerically calculated, in terms of $T_{x}$ and $R_{x}$, as

$$
\mu=\frac{1.8}{\left(1+2 T_{x}\right)\left(1+2 R_{x}\right)} .
$$

For supporting the accuracy of this estimation, in Fig. 2 . (9) is compared to the the channel statistics of $\mathbf{C}$ for $N=1$, which is obtained through the Monte Carlo simulations performing at least $10^{6}$ trials for each $R_{x} \times T_{x}$ set-up. The results show that the estimated $\mu$ in $(9)$ perfectly fit the computer simulations per $R_{x} \times T_{x}$.

Then, 9 is utilized to determine the ABEP of the system in the following subsection.

\section{B. ABEP Analysis}

In this subsection, after obtaining a numerical approximation for the statistics of the channel matrix $\mathbf{C}$, an upper bound expression for the ABEP of the proposed system is given as follows [18]:

$$
P_{e} \leq \frac{1}{\kappa 2^{\kappa}} \sum_{\mathbf{x}} \sum_{\hat{\mathbf{x}}} P(\mathbf{x} \rightarrow \hat{\mathbf{x}}) e(\mathbf{x}, \hat{\mathbf{x}})
$$

where $\kappa$ is the number of incoming information bits, $P(\mathbf{x} \rightarrow$ $\hat{\mathbf{x}})$ is the unconditional pairwise error probability (PEP) and $e(\mathbf{x}, \hat{\mathbf{x}})$ is the number of error bits for the corresponding PEP event.

To obtain the PEP expression, first, the conditional PEP (CPEP) of the system is derived, using the $Q$-function, as follows

$$
P(\mathbf{x} \rightarrow \hat{\mathbf{x}} \mid \mathbf{C})=Q\left(\sqrt{\frac{\Omega}{2 \sigma^{2}}}\right)
$$


where $\Omega$ is given, for $\boldsymbol{\Delta}=(\mathbf{x}-\hat{\mathbf{x}})(\mathbf{x}-\hat{\mathbf{x}})^{H}$, as

$$
\begin{aligned}
\Omega & =\|\mathbf{C}(\mathbf{x}-\hat{\mathbf{x}})\|^{2} \\
& =\operatorname{vec}\left(\mathbf{C}^{\mathrm{H}}\right)^{\mathrm{H}}\left(\boldsymbol{\Delta} \otimes \mathbf{I}_{R_{x}}\right) \operatorname{vec}\left(\mathbf{C}^{\mathrm{H}}\right) .
\end{aligned}
$$

Therefore, considering $Q(x)=\frac{1}{\pi} \int_{0}^{\pi / 2} e^{-x^{2} / 2 \sin ^{2} \theta} d \theta$, the CPEP (11) can be rewritten as

$$
\begin{aligned}
& P(\mathbf{x} \rightarrow \hat{\mathbf{x}} \mid \mathbf{C})= \\
& \quad \frac{1}{\pi} \int_{0}^{\pi / 2} \exp \left(-\varphi \frac{\operatorname{vec}\left(\mathbf{C}^{\mathrm{H}}\right)^{\mathrm{H}}\left(\boldsymbol{\Delta} \otimes \mathbf{I}_{R_{x}}\right) \operatorname{vec}\left(\mathbf{C}^{\mathrm{H}}\right)}{4 \sin ^{2} \theta}\right) d \theta
\end{aligned}
$$

where $\varphi=1 / N_{0}$. Then, averaging 14 over the matrix $\mathbf{C}$ through moment generating function (MGF) approach results in the following PEP expression

$$
P(\mathbf{x} \rightarrow \hat{\mathbf{x}})=\frac{1}{\pi} \int_{0}^{\pi / 2} M_{\Omega}\left(\frac{-\varphi}{4 \sin ^{2} \theta}\right) d \theta
$$

In [19], the MGF of $\mathbf{z}^{\mathrm{H}} \mathbf{B z}$, for any Hermitian $\mathbf{B}$ matrix, is given by

$$
M(s)=\exp \left(\frac{s \overline{\mathbf{z}}^{\mathrm{H}} \mathbf{B}\left(\mathbf{I}-s \mathbf{C}_{\mathbf{z}} \mathbf{B}\right)^{-1} \overline{\mathbf{z}}}{\operatorname{det}\left(\mathbf{I}-s \mathbf{C}_{\mathbf{z}} \mathbf{B}\right)}\right)
$$

where $\overline{\mathbf{z}}$ is the mean vector and $\mathbf{C}_{\mathbf{z}}$ is the covariance matrix of the vector $\mathbf{z}$.

Clearly, in our system $\mathbf{B}=\boldsymbol{\Delta} \otimes \mathbf{I}_{R_{x}}$ and $\mathbf{z}=\operatorname{vec}\left(\mathbf{C}^{\mathrm{H}}\right)$. Using the numerically obtained channel statistics given in the previous subsection, $\overline{\mathbf{z}}=\overline{\operatorname{vec}\left(\mathbf{C}^{\mathrm{H}}\right)}=\mu N \mathbf{1}$ and $\mathbf{C}_{\mathbf{z}}=N \mathbf{I}$ are respectively defined as the mean vector and the covariance matrix for the Gaussian vector $\mathbf{z}=\operatorname{vec}\left(\mathbf{C}^{\mathrm{H}}\right)$. Therefore, the PEP of the system becomes

$$
\begin{aligned}
& P(\mathbf{x} \rightarrow \hat{\mathbf{x}})= \\
& \frac{1}{\pi} \int_{0}^{\pi / 2} \frac{\exp \left(-\overline{\mathbf{z}}^{\mathrm{H}} \frac{\varphi}{4 \sin ^{2} \theta} \mathbf{B}\left(\mathbf{I}+\frac{\varphi}{4 \sin ^{2} \theta} \mathbf{C}_{\mathbf{z}} \mathbf{B}\right)^{-1} \overline{\mathbf{z}}\right)}{\operatorname{det}\left(\mathbf{I}+\frac{\varphi}{4 \sin ^{2} \theta} \mathbf{C}_{\mathbf{z}} \mathbf{B}\right)} d \theta .
\end{aligned}
$$

\section{Simulation Results}

In this section, the BER performance of the proposed RIS-MIMO and RIS-SM schemes are investigated through theoretical analysis and comprehensive computer simulations. All results are performed as a function of transmitted signal energy to noise ratio $\left(E_{s} / N_{0}\right)$ and for different $R_{x} \times T_{x}$ MIMO configurations and BPSK modulation $(M=2)$.

In Fig. 3, for $4 \times 2$ MIMO and various $N$ reflecting elements, the theoretical BER performance of RIS-MIMO and RIS-SM schemes are compared with the computer simulation results. It is obvious from this figure that the derived semianalytic results perfectly match with the simulation results as $N$ increases.

In Fig. 4, for $4 \times 4$ system configuration, the BER performance of the RIS-MIMO and RIS-SM schemes using the proposed cosine similarity theorem-based algorithm and pseudoinverse (pinv)-based algorithm [9] is compared. The results show that compared to [9], the BER performance of both RIS-MIMO and RIS-SM schemes using the proposed algorithm, which requires significantly lower computational complexity, improves better as $N$ increases.

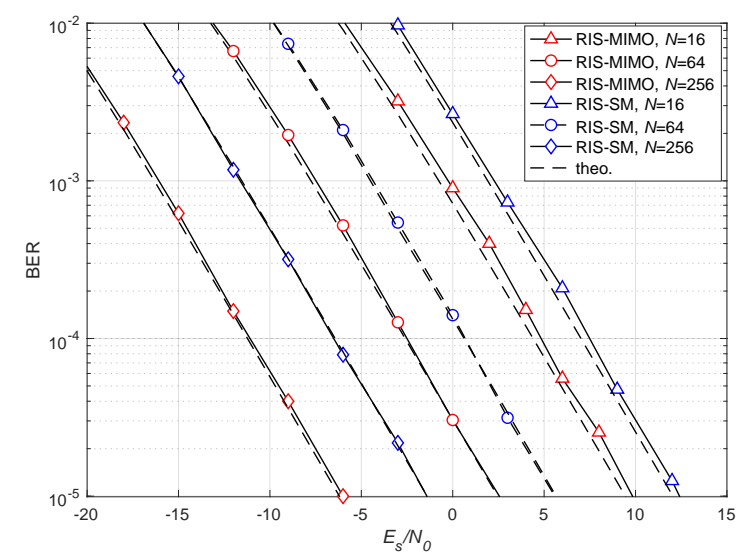

Fig. 3. Theoretical and simulation results of the RIS-MIMO ve RIS-SM schemes.

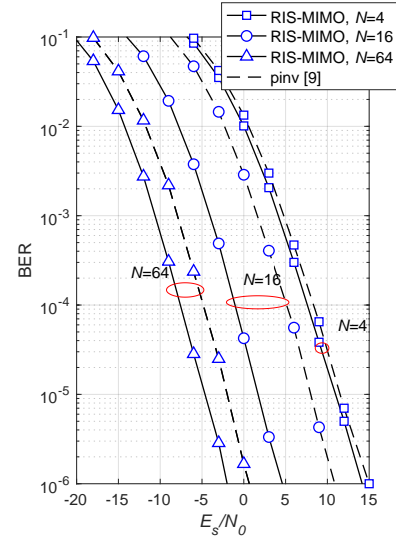

(a)

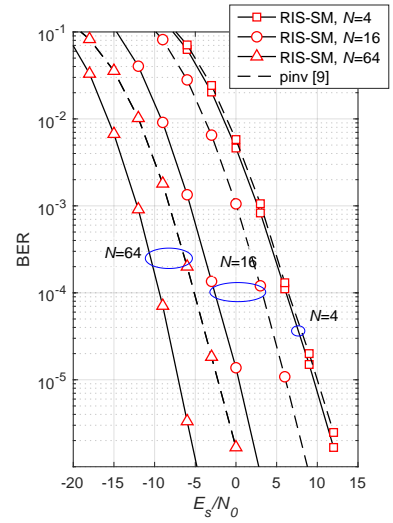

(b)
Fig. 4. Comparison of the proposed algorithm to the pinv algoritm [9] on the BER performance of the a) RIS-MIMO and b) RIS-SM schemes.

As an illustration, the proposed algorithm with $\mathcal{O}\left(6 N\left(T_{x}+\right.\right.$ $\left.R_{x}\right)$ ) complexity performs $3072 \mathrm{RMs}$ for $N=64$, while the reference pinv algorithm [9] with $\mathcal{O}\left(N^{3}\right)$ complexity performs 262144 RMs. This means, the proposed algorithm provides $98.8 \%$ reduction in computational complexity over the reference algorithm [9] for $N=64$.

In Fig. 5, the impact of the imperfect channel knowledge and the use of discrete phase shifts on the BER performance of the $4 \times 4$ RIS-MIMO scheme is investigated for $N$ reflecting elements.

For discrete phase shifts model, we assume that each reflecting element at the RIS takes a finite number of discrete phase values. Therefore, a set of discrete phase shifts is constructed by uniformly quantizing $[0, \pi]^{2}$ interval into $N$ levels as $\mathcal{F}=\{0, \Delta \phi, \ldots,(N-1) \Delta \phi\}$, where $\Delta \phi=\pi / N$. Then, the continous phase shift of the each reflecting element, which is obtained via the proposed algorithm, is rounded to the nearest discrete level. The simulation results demonstrate that discrete phase reflection causes a minor BER performance degradation compared to the continous phase reflection. However, this performance difference gradually disapperars as $N$ increases.

Moreover, to analyze the effect of the channel estimation error, the imperfect CSI is assumed at both the RIS and the receiver. Therefore, we consider that both the RIS and the

${ }^{2}$ In Algorithm I, for $\arccos (\cdot)$ operation, MATLAB function $\operatorname{acos}()$ is used, which returns phase values between the interval $[0, \pi]$. 


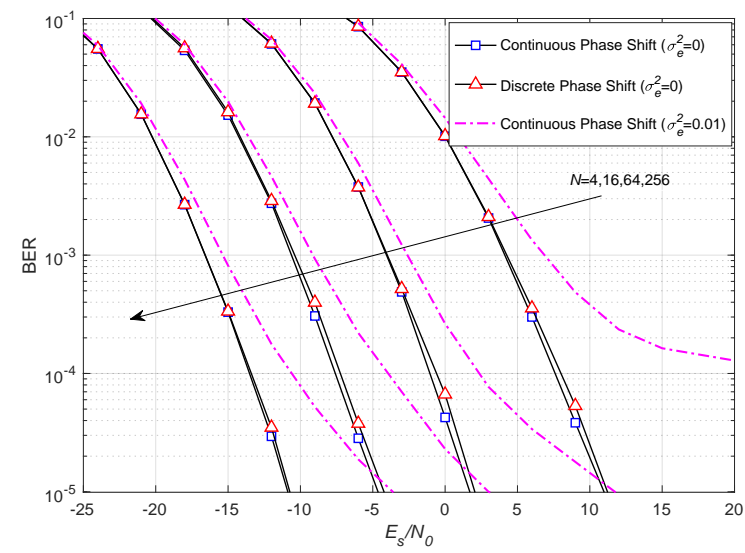

Fig. 5. BER Performance of RIS-MIMO scheme with discrete and continous phase reflection for the channel estimation error variance $\sigma_{e}^{2}$.

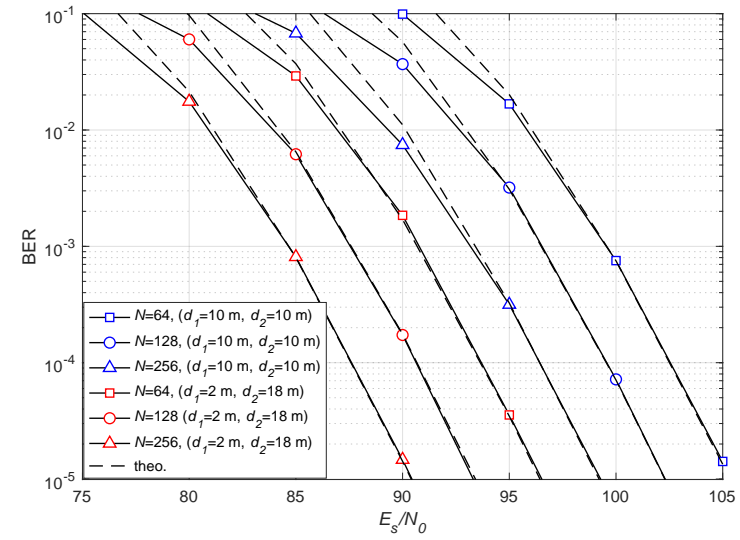

Fig. 6. BER Performance of RIS-MIMO scheme in case of the path loss effect [10].

receiver errorneously estimate $\mathbf{H}$ and $\mathbf{G}$ channel matrices as $\overline{\mathbf{H}} \in \mathbb{C}^{N \times T_{x}}=\mathbf{H}+\mathbf{E}_{\mathbf{t}}$ and $\overline{\mathbf{G}} \in \mathbb{C}^{N \times R_{x}}=\mathbf{G}+\mathbf{E}_{\mathbf{r}}$, respectively, where $\mathbf{E}_{\mathbf{t}}$ and $\mathbf{E}_{\mathbf{r}}$ are the matrices of the channel estimation errors whose each entry is i.i.d. and distributed as $\mathcal{C N}\left(0, \sigma_{e}^{2}\right)$. Therefore, after the proposed algorithm determines the diagonal reflection matrix $\overline{\boldsymbol{\Phi}} \in \mathbb{C}^{N \times N}$ considering the imperfect channels $\overline{\mathbf{H}}$ and $\overline{\mathbf{G}}$, the overall estimated channel matrix becomes $\overline{\mathbf{C}} \in \mathbb{C}^{R_{x} \times T_{x}}=\overline{\mathbf{G}}^{\mathrm{H}} \overline{\mathbf{\Phi}} \overline{\mathbf{H}}$. The simulation results reveal that the assumption of the imperfect CSI at both the RIS and the receiver significantly degrades the BER performance of the RIS-MIMO scheme. However, increasing the number of reflecting elements at the RIS reduces this performance degradation.

Fig. 6 demonstrates the BER performance of the proposed RIS-MIMO scheme with $4 \times 4$ when path loss effect [10] is considered. Denoting the distances from the transmitter to the RIS and from the RIS to the receiver as $d_{1}$ and $d_{2}$, respectively, the path loss $P_{L}$ of the overall system is calculated as [10]

$$
P_{L}^{-1}=\frac{\lambda^{4}}{256 \pi^{2}} \frac{1}{d_{1}^{2} d_{2}^{2}}
$$

where $\lambda$ is the wavelength at $2.4 \mathrm{GHz}$ operating frequency. The simulations are performed for $d_{1}=d_{2}=10 \mathrm{~m}$ and $d_{1}=2 \mathrm{~m}$, $d_{2}=18 \mathrm{~m}$. It can be deduced from the results that compared to the performance in the absence of the path loss given in Fig. 4, the error performance of the RIS-MIMO scheme significantly degrade with the path loss effect. On the other hand, since the
$P_{L}$ is proportional to $d_{1}^{2} d_{2}^{2}$, the BER performance of the system fairly improves when the RIS is closer to the transmitter or the receiver $\left(d_{1}=2 \mathrm{~m}\right.$ and $\left.d_{2}=18 \mathrm{~m}\right)$.

\section{CONCLUSION}

In this letter, a low-complexity algorithm has been developed exploiting the cosine similarity theorem for RIS aided MIMO transmission schemes to enhance the overall path gain of the communication channel. Moreover, performing a semianalytic approach, the ABEP of the system has been derived. Furthermore, through the comprehensive computer simulations the BER performance of RIS aided MIMO schemes for different circumstances including discrete phase reflection, path loss effect and imperfect CSI has been investigated.

\section{REFERENCES}

[1] S. Zhang and R. Zhang, "Capacity characterization for intelligent reflecting surface aided MIMO communication," arXiv preprint arXiv:1910.01573, 2019.

[2] Q.-U.-A. Nadeem, A. Kammoun et al., "Large intelligent surface assisted MIMO communications," arXiv preprint arXiv:1903.08127, 2019.

[3] E. Basar, "Transmission through large intelligent surfaces: A new frontier in wireless communications," in IEEE European Conf. Netw. and Commun. (EuCNC), June 2019, pp. 112-117.

[4] E. Basar, M. Di Renzo et al., "Wireless communications through reconfigurable intelligent surfaces," IEEE Access, vol. 7, pp. $116753-$ 116773, Aug. 2019.

[5] E. Basar, "Reconfigurable intelligent surface-based index modulation: A new beyond MIMO paradigm for 6G," IEEE Trans. Commun., Feb. 2020.

[6] S. Gopi, S. Kalyani, and L. Hanzo, "Intelligent reflecting surface assisted beam index-modulation for millimeter wave communication," arXiv preprint arXiv:2003.12049, 2020.

[7] C. Huang, A. Zappone et al., "Reconfigurable intelligent surfaces for energy efficiency in wireless communication," IEEE Tran. Wireless Commun., vol. 18, no. 8, pp. 4157-4170, June 2019.

[8] Q. Wu and R. Zhang, "Intelligent reflecting surface enhanced wireless network via joint active and passive beamforming," IEEE Trans. Wireless Commun., vol. 18, no. 11, pp. 5394-5409, Aug. 2019.

[9] T. Hou, Y. Liu et al., "MIMO assisted networks relying on large intelligent surfaces: A stochastic geometry model," arXiv preprint arXiv:1910.00959, 2019.

[10] S. W. Ellingson, "Path loss in reconfigurable intelligent surface-enabled channels," arXiv preprint arXiv:1912.06759, 2019.

[11] W. Tang, M. Z. Chen et al., "Wireless communications with reconfigurable intelligent surface: Path loss modeling and experimental measurement," arXiv preprint arXiv:1911.05326, 2019.

[12] E. Basar, I. Yildirim, and I. F. Akyildiz, "Indoor and outdoor physical channel modeling and efficient positioning for reconfigurable intelligent surfaces in mmWave bands," arXiv preprint arXiv:2006.02240, 2020.

[13] _ _SimRIS channel simulator for reconfigurable intelligent surfaceempowered communication systems," arXiv preprint arXiv:2006.00468, 2020.

[14] S. Abeywickrama, R. Zhang et al., "Intelligent reflecting surface: Practical phase shift model and beamforming optimization," arXiv preprint arXiv:2002.10112, 2020.

[15] Q. Wu and R. Zhang, "Weighted sum power maximization for intelligent reflecting surface aided SWIPT," IEEE Wireless Commun. Lett., vol. 9, no. 5, pp. 586-590, 2019.

[16] T. K. Moon and W. C. Stirling, Mathematical Methods and Algorithms for Signal Processing. Prentice Hall Upper Saddle River, NJ, 2000, vol. 1.

[17] R. Y. Mesleh, H. Haas et al., "Spatial modulation," IEEE Trans. Veh. Technol., vol. 57, no. 4, pp. 2228-2241, July 2008.

[18] M. K. Simon and M.-S. Alouini, Digital communication over fading channels. John Wiley \& Sons, 2005, vol. 95.

[19] G. L. Turin, "The characteristic function of hermitian quadratic forms in complex normal variables," Biometrika, vol. 47, no. 1/2, pp. 199-201, 1960. 